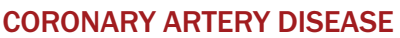

\section{Darapladib fails to improve the STABILITY of CAD}

The potent and selective oral inhibitor of lipoprotein-associated phospholipase $\mathrm{A}_{2}\left(\mathrm{Lp}-\mathrm{PLA}_{2}\right.$ ) darapladib does not reduce the rate of ischeamic events in patients with coronary heart disease (CHD). This finding from the placebo-controlled STABILITY trial was presented at the ACC 2014 Scientific Sessions and simultaneously published in the New England Journal of Medicine. "The STABILITY trial is the first large-scale, randomized, global trial to test a novel mechanism of inhibition of inflammation in atherosclerotic plaque," explains co-chair of the study Dr Harvey White.

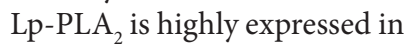
vulnerable atherosclerotic plaque, and Lp-PLA 2 activity has been shown to be associated with CHD risk. Preclinical and early clinical studies of darapladib showed promising reductions in Lp-PLA and slowed the progression of atherosclerosis. The stage was, therefore, set for a large, phase III trial to investigate the effects of darapladib on 'hard' clinical end points.

The STABILITY trial population comprised 15,828 patients from 39 countries. All participants had documented CHD and one additional predictor of cardiovascular risk. Patients were typically male (81\%), white (78\%), and elderly (median age 65 years). Almost $60 \%$ of trial participants had experienced a prior myocardial infarction, and $75 \%$ had undergone coronary revascularization. Importantly, the use of guidelinerecommended therapies was high; $97 \%$ of patients received a statin and $93 \%$ were taking aspirin.

During follow-up (median 3.7 years), the composite primary end point (cardiovascular death, myocardial infarction, or stroke) occurred in $9.7 \%$ of the 7,924 patients randomly assigned to darapladib and $10.4 \%$ of the 7,904 patients in the placebo group (HR 0.94, 95\% CI 0.85-1.03, $P=0.20)$. None of the individual components of this end point were significantly reduced by darapladib compared with placebo. However, a reduction in the first prespecified secondary end point (composite of major coronary events) occurred with darapladib that just reached statistical significance (HR 0.90, 95\% CI 0.82-1.00, $P=0.045$ ).

"We believe there is a signal on coronary events with about a $10 \%$ lower rate," says Dr White. "These events are important for patients."

Notably, more patients in the darapladib group discontinued the drug than in the placebo group, predominantly owing to unpleasant adverse effects such as diarrhoea.

However, more worringly, the rate of renal dysfunction was higher in patients who received darapladib than placebo.

The STABILITY investigators postulate that darapladib might have less of an effect on atherosclerotic plaque than was thought previously, or that the excellent background therapy received by the study participants left little scope for additional benefit from darapladib. Nevertheless, the ongoing phase III SOLID-TIMI 52 trial of darapladib in acute coronary syndromes will provide additional information on the efficacy of this drug, and determine whether the development of darapladib continues.

\section{Alexandra Roberts}

Original article The STABILITY investigators. Darapladibfor preventing ischemic events in stable coronary heart disease. N. Engl. J. Med. doi:10.1056/NEJMoa1315878

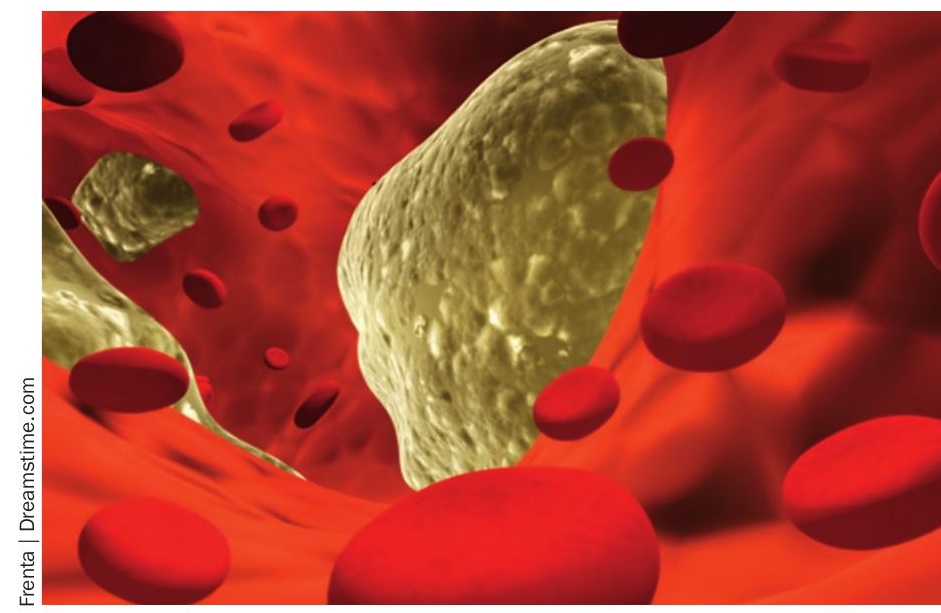

